ARTICLE

Received 19 Aug 2013 | Accepted 10 Jan 2014 | Published 21 Feb 2014 DOl: 10.1038/ncomms4247

\title{
Linking experiment and theory for three-dimensional networked binary metal nanoparticle-triblock terpolymer superstructures
}

Zihui $\mathrm{Li}^{1}{ }^{1 \star}$, Kahyun Hur ${ }^{2, \star}, \dagger$, Hiroaki Sai ${ }^{2}$, Takeshi Higuchi ${ }^{3}$, Atsushi Takaharaa ${ }^{3,4}$, Hiroshi Jinnai ${ }^{3,4}$, Sol M. Gruner ${ }^{5,6}$ \& Ulrich Wiesner ${ }^{2}$

Controlling superstructure of binary nanoparticle mixtures in three dimensions from selfassembly opens enormous opportunities for the design of materials with unique properties. Here we report on how the intimate coupling of synthesis, in-depth electron tomographic characterization and theory enables exquisite control of superstructure in highly ordered porous three-dimensional continuous networks from single and binary mixtures of metal nanoparticles with a triblock terpolymer. Poly(isoprene-block-styrene-block-(N,Ndimethylamino)ethyl methacrylate) is synthesized and used as structure-directing agent for ligand-stabilized platinum and gold nanoparticles. Quantitative analysis provides insights into short- and long-range nanoparticle-nanoparticle correlations, and local and global contributions to structural chirality in the networks. Results provide synthesis criteria for next-generation mesoporous network superstructures from binary nanoparticle mixtures for potential applications in areas including catalysis.

\footnotetext{
${ }^{1}$ Department of Chemistry and Chemical Biology, Cornell University, Ithaca, New York 14853, USA. ${ }^{2}$ Department of Materials Science and Engineering, Cornell University, Ithaca, New York 14853, USA. ${ }^{3}$ Japan Science and Technology Agency, ERATO, Takahara Soft Interfaces Project and Institute for Materials Chemistry and Engineering (IMCE), CE80, Kyushu University, 744 Motooka, Nishi-ku, Fukuoka 819-0395, Japan. ${ }^{4}$ International Institute for Carbon-Neutral Energy Research (WPI-12CNER), CE80, Kyushu University, 744 Motooka, Nishi-ku, Fukuoka 819-0395, Japan. ${ }^{5}$ Department of Physics, Cornell University, Ithaca, New York 14853, USA. ${ }^{6}$ Cornell High Energy Synchrotron Source, Cornell University, Ithaca, New York 14853, USA. ${ }^{\star}$ These authors contributed equally to this work. †Present address: Institute of Multidisciplinary Convergence of Materials, Korea Institute of Science and Technology, Seoul 136-791, Republic of Korea. Correspondence and requests for materials should be addressed to U.W. (email: ubw1@cornell.edu).
} 
C ontrolling the spatial arrangement of metal and semiconductor nanoparticles (NPs) from bottom-up selfassembly remains a long-standing scientific goal in materials synthesis ${ }^{1-10}$. Assembly of NPs as artificial 'atoms' into ordered structures provides facile access to a variety of previously unknown nanomaterials. Effective control over electrostatic and entropic interactions between NPs leads to highly ordered materials with structural diversity ${ }^{2-5,9}$. Complementary molecules, for example, DNA ${ }^{1,9,10}$ or block copolymers (BCPs) ${ }^{11}$, attached to NPs mediate their ordered assemblies enabling programmable structure control. Despite this progress, major challenges remain in controlling the structures of metal and semiconductor NP assemblies at the mesoscale-for example, to provide mesoporosity as well as three-dimensional (3D) connectivity, highly desirable for applications requiring efficient charge transport in electrodes or electrocatalytic materials ${ }^{12-14}$

In particular, highly ordered mesoporous 3D bicontinuous network superstructures of metal NPs have stayed elusive, especially from mixtures of different NPs. Such 3D network structures, particularly if they are mesoporous, are of specific interest as they are expected to provide excellent mechanical stability, high accessibility to NP surface area and high connectivity.

Here, we demonstrate how the combination of controlled NP synthesis, detailed structural analysis by transmission electron microtomography $(\mathrm{TEMT})^{15}$, energy-dispersive X-ray spectroscopy (EDS) and percolation theory, as well as comparison with a recently developed self-consistent field theory (SCFT) ${ }^{16,17}$ enabled highly ordered and porous 3D network formation of single and binary metal NPs from triblock terpolymer self-assembly with particle locations that can be rationalized. Analysis further provided insights into short- and long-range NP-NP correlations, and local and global contributions to structural chirality in an alternating gyroid $\left(\mathrm{G}^{\mathrm{A}}\right)$ domain.

\section{Results}

Alternating gyroid structure. Gyroid structures have been observed in low molar mass surfactant-water systems and BCP self-assembly ${ }^{18,19}$ based on the minimal gyroid surface (Schoen's G-surface, symmetry $I a \overline{3} d)^{20}$. Structures in these systems usually consist of two intertwining gyroid subvolumes related by an inversion operation. If these two subvolumes are constituted by the same material, the structure is a double gyroid $\left(\mathrm{G}^{\mathrm{D}}, I a \overline{3} d\right)$. Each gyroid network contains chiral helices along various crystallographic directions. In the $\mathrm{G}^{\mathrm{D}}$ local chirality is cancelled, since respective screw axes of each gyroid network are of opposite handedness. The alternating (single) gyroid $\left(\mathrm{G}^{\mathrm{A}}\right.$, / $4_{1} 32$ ), in contrast, contains two subvolumes of different materials, thus exhibiting local chirality. The $\mathrm{G}^{\mathrm{A}}$ structure is found in several tropical butterfly species and depicts a unique chiral 3D periodic morphology ${ }^{21}$. While the periodic $\mathrm{G}^{\mathrm{D}}$ network structure is accessible via di-BCP self-assembly, the $G^{A}$ structure is only accessible via triblock terpolymers, which provides three distinct periodic block volumes. Triply periodic network phases formed by triblock terpolymers also form over much wider composition windows $(4-14 \mathrm{vol} \%)^{22,23}$ than the $\mathrm{G}^{\mathrm{D}}$ structure found in $\mathrm{di}$ BCPs $(2-6 \text { vol\% })^{24,25}$. Furthermore, mesoporous materials derived from the $\mathrm{G}^{\mathrm{A}}$ phase typically have larger pore volume than those from the $\mathrm{G}^{\mathrm{D}}$ phase. Finally, we also note that the chiral nature of the $\mathrm{G}^{\mathrm{A}}$ structure can lead to interesting properties ${ }^{26}$.

Synthesis of terpolymer and hybrids. Precise control of spatial organization of ligand-stabilized platinum $(\mathrm{Pt})$ and gold $(\mathrm{Au})$ NPs was achieved with a novel triblock terpolymer, poly (isoprene-block-styrene-block-(N,N-dimethylamino)ethyl methacrylate) (PI- $b$-PS- $b$-PDMAEMA), which will be referred to as ISA, (Fig. 1a-c). Polymer ISA had a molar mass of $102,300 \mathrm{~g} \mathrm{~mol}^{-1}$, a poydispersity index below 1.1 , and a composition of I, S and A of 23.4, 71.5 and $5.1 \mathrm{wt} \%$, respectively (see Methods and Supplementary Fig. 1a). Ligand-stabilized Pt NPs were synthesized by an improved synthesis scheme, while Au NPs were prepared according to known methods; see Methods. Ar-O plasma etching of organics resulted in $3 \mathrm{D}$ mesoporous metal networks comprising one or two different types of metal NPs.

SAXS characterization of hybrid films. With the synthesis of the ISA triblock terpolymer and careful choice of the NP ligands, we obtained networked hybrid materials with Pt metal NPs as well as with binary mixtures of $\mathrm{Pt}$ and $\mathrm{Au}$ metal NPs (see Table 1 for hybrid compositions). We first characterized the NP superstructures by small-angle X-ray scattering (SAXS). Figure 1e compares SAXS results of the parent ISA and the resulting hybrids obtained by polymer NP mixing in a common solvent and subsequent casting of films (see Methods). Neat ISA showed a microphase-separated hexagonal morphology as suggested by SAXS and transmission electron microscopy (TEM; Fig. 1e and Supplementary Fig. 1b).

Hybrid ISA-H1 with $23.1 \mathrm{wt} \%$ Pt NPs exhibited a scattering curve containing up to 10 higher-order peaks, very different from the parent polymer trace. Tick marks in Fig. 1e for this scaling trace show expected peak positions for a $\mathrm{G}^{\mathrm{A}}$ lattice with $I 4_{1} 32$ space group, with $\left(q / q_{100}\right)^{2}=2,6,8,10,12,14,16,18,20,22,24$, $26,30,32,34$, where the scattering vector $q$ is defined by $q=4$ $\pi \sin \theta / \lambda$, with scattering angle $2 \theta$, X-ray wavelength $\lambda$ and reciprocal vector, $q_{100}$, describing the cubic lattice parameter. Expected and observed peak positions match well, suggesting that the SAXS trace of the hybrid is consistent with the $G^{A}$ morphology. The narrow first-order peak and clear higher-order peaks further suggest well-defined interfaces and large grain sizes. Addition of Pt NPs to ISA thus resulted in a phase transformation from a hexagonal to a cubic network $\left(G^{A}\right)$ structure. We further increased the loading of Pt NPs resulting in lamellar morphology as predicted from theory ${ }^{17}$ (see Supplementary Fig. 1c for the morphology map of ISA-Pt NP hybrids).

TEM characterization of hybrid films. The assignment of hybrid morphology was corroborated by TEM characterization. Figure $2 \mathrm{a}-\mathrm{f}$ shows representative TEM images of $100-\mathrm{nm}$-thick microtomed sections of hybrid ISA-H1 exhibiting different projections. The metal NP + PDMAEMA domains appear dark providing image contrast to the PI and PS domains. Fourfold (100) and threefold (111) projections (Fig. 2a,b) characteristic for a cubic structure can clearly be identified. Other planes, for example (110) and (210) planes, are also often observed in TEM, see Fig. 2c,d. Insets in Fig. 2a-d represent NP network images of the respective $\mathrm{G}^{\mathrm{A}}$ projection obtained from SCFT simulations, which match well with the experimental data. Quantitative analysis of TEM images stays challenging, however, as information of a $3 \mathrm{D}$ structure is reduced to a two-dimensional (2D) plane and because of possible PI domain distortions in microtomed thin slices originating from the low $T_{\mathrm{g}}$ of PI $\left(-60^{\circ} \mathrm{C}\right)$. Indeed, the cubic lattice parameters, $a$, calculated from these images are all between 50 and $60 \mathrm{~nm}$, that is, slightly underestimating the value of $63 \mathrm{~nm}$ from SAXS. Higher magnification TEM images of ISA$\mathrm{H} 1$ (Fig. 2e) resolve individual Pt NPs filling the struts of the network structure. Staining PI domains with $\mathrm{OsO}_{4}$ provided additional contrast to the material and enabled simultaneous visualization of struts of the two gyroid minority networks. Figure $2 \mathrm{f}$ shows the TEM image of a (111) projection of ISA-H1 where PI domains appear in light grey and Pt NP + PDMAEMA domains in dark. The image clearly reveals that the two domains 
a

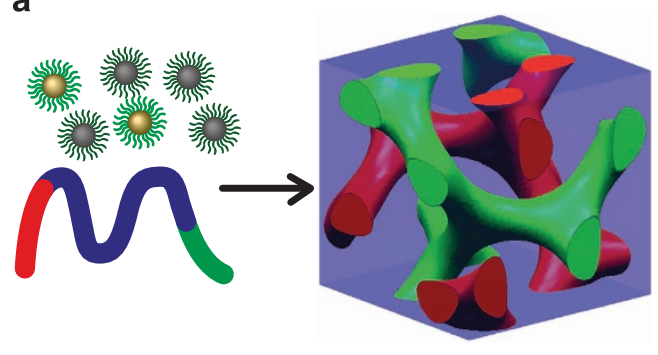

b

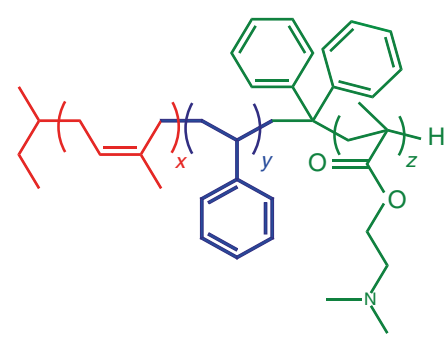

C

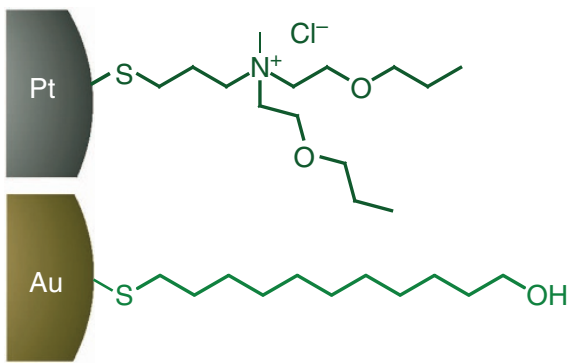

d

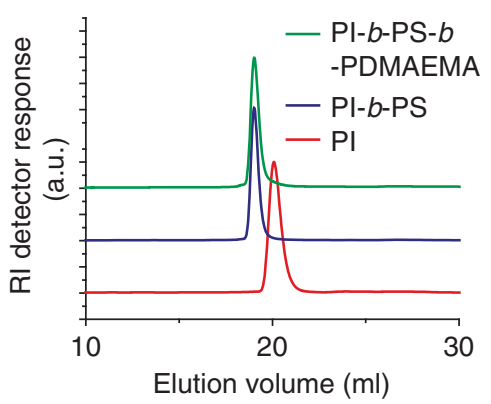

e

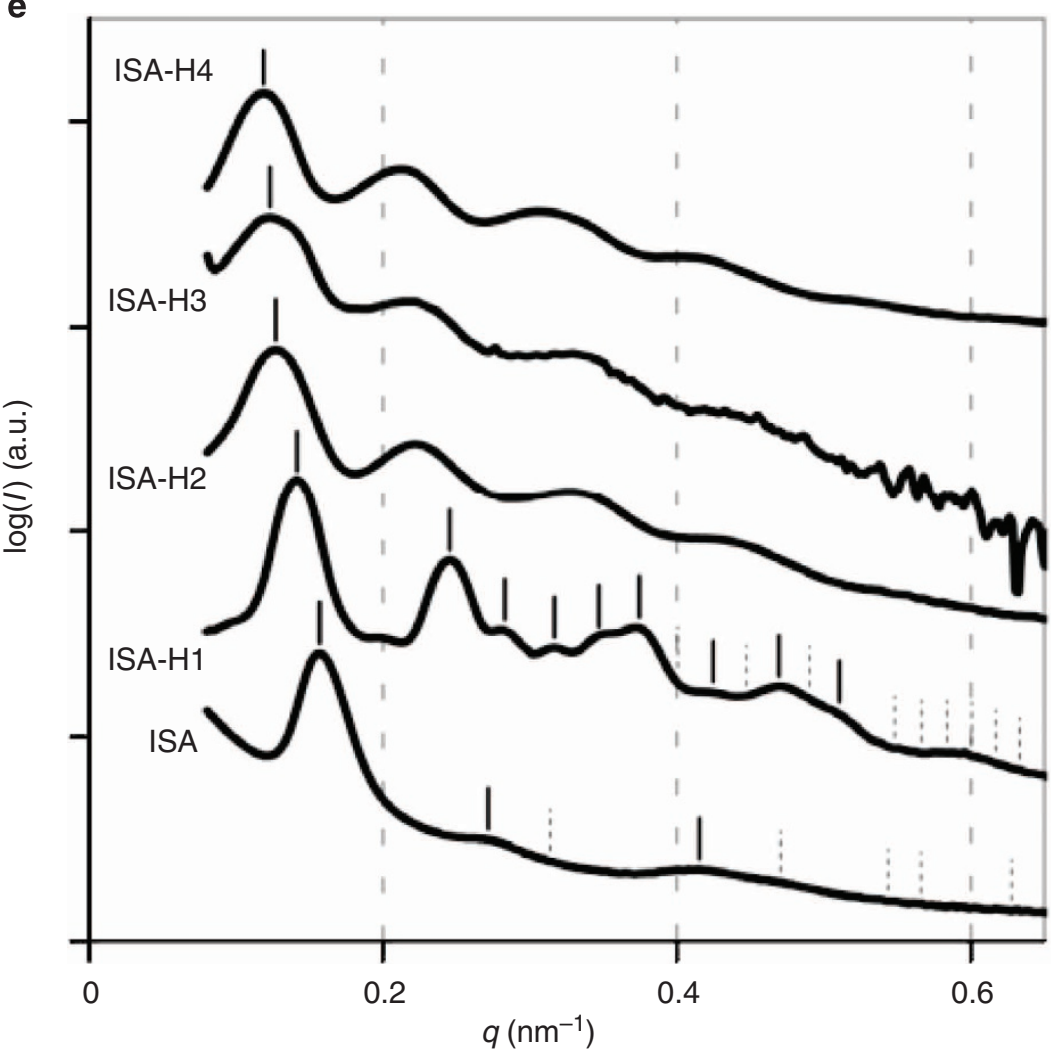

Figure 1 | Hybrid synthesis components and schematic as well as characterization results. (a) Scheme of triblock terpolymer-binary Pt and Au NPs selfassembly; (b) molecular structure of PI-b-PS-b-PDMAEMA (ISA); (c) molecular structure of ligands attached to Pt, N,N-di-(2-(allyloxy)ethyl)-N-3mercaptopropyl- $\mathrm{N}$-methylammonium chloride and Au NPs, 11-mercapto-1-undecanol; (d) size exclusion chromatograph data for different triblock terpolymer synthesis steps; (e) from bottom to top SAXS data of neat polymer ISA, Pt NP hybrid ISA-H1, binary Pt and Au NP hybrids ISA-H2, ISA-H3 and ISA-H4 with increasing Au NP content. Tick marks show expected peak positions for hexagonal (ISA) and GA (ISA-H1) structures. Solid and dotted tick marks distinguish between observed and missing peaks for ISA and ISA-H1, respectively. The tick mark positions for ISA-H2, ISA-H3 and ISA-H4 are used for determining the lattice spacing $a$ in Table 1.

form two networks that are intertwining with each other. Figure $2 \mathrm{~b}, \mathrm{f}$ also shows the triple node connectivity of the material, which is characteristic of gyroidal structures.
Mesoporous film formation. To maintain the superstructure on removal of the structure-directing polymer on the way to mesoporous materials, NP percolation in all three directions is needed. 
Table 1 | Terpolymer-binary metal NP hybrid compositions.

\begin{tabular}{lccc} 
& $\begin{array}{c}\text { Total NP in total } \\
\text { mass (wt\%) }\end{array}$ & $\begin{array}{c}\text { Au in total } \\
\text { NP (wt\%) }\end{array}$ & $\boldsymbol{a}(\mathbf{n m})$ \\
\hline ISA-H1 & 23.1 & 0 & 62.8 \\
ISA-H2 & 18.2 & 5 & 69.8 \\
ISA-H3 & 19.5 & 10 & 71.7 \\
ISA-H4 & 19.2 & 15.8 & 74.6 \\
\hline
\end{tabular}

Despite a decrease of total NP content, a systematic increase of $a$ with increasing Au NP content is observed, where $a$ is the cubic lattice parameter obtained from SAXS.

A percolation analysis shows that ISA-H1 is very close to a NP percolation threshold ${ }^{27}$ suggesting that the superstructure may survive ISA removal (see 'NP percolation' paragraph in Methods section and Supplementary Fig. 2). Ar-O plasma treatment of $100 \mathrm{~nm}$ slices of as-made hybrids was used to test this hypothesis. The high quality structure control in the resulting porous NP superstructures is revealed in the representative TEM image of sample ISA-H1 in Fig. $2 \mathrm{i}$ showing the boundary between two grains running diagonally through the middle of the image. The individual Pt NPs constituting the superstructure can clearly be distinguished in the higher magnification image in the inset of Fig. 2i. EDS data indicate that a large fraction of organic materials including the ligands were etched away during the plasma treatment; see Supplementary Fig. 3. However, because of the low $\mathrm{X}$-ray fluorescence yield of carbon atoms, quantification of organic content after plasma treatment has a rather large error bar associated with it. From the spectra in Supplementary Fig. 3c alone, it is thus not possible to exclude remaining organic material. The porous NP superstructure survived the plasma treatment probably because of NP percolation mediated by shrinkage of the gyroidal network while removing organic materials (see 'NP percolation' paragraph in Methods section).

3D local, global and chiral structures characterization. A 3D TEMT reconstruction provides visualization of the 3D nanostructure in real space at a resolution of a few Angstroms, high enough to distinguish individual NPs ${ }^{15}$. Reconstruction of the 3D superstructure of as-made hybrid ISA-H1, that is, without the plasma treatment, was chosen for $\mathrm{G}^{\mathrm{A}}$ structure analysis owing to the best long-range order among all hybrids. Results are shown in Fig. 3 where red spheres represent the Pt NP cores. Since NPs are coated with ligands, most of the NP cores are separated (note that identification of organic parts of the hybrid, that is, polymers and ligands, is challenging because of their small electron density contrast; see also Fig. 2). From reconstruction of NP cores the 3D superstructure is not easily identified. This could be achieved by increasing the NP diameters, that is, by convoluting the NP cores with a sphere of diameter $d$. The resulting overlapping volumes for the case of $d=6.5 \mathrm{~nm}$ are shown via the green surfaces in Fig. 3, clearly giving rise to a simple network structure. To further demonstrate that this network is consistent with the $G^{A}$ morphology, we compared it with a superimposed $\mathrm{G}^{\mathrm{A}}$ network obtained from a SCFT simulation; see the bottom right of Fig. 3.

Chirality of $\mathrm{G}^{\mathrm{A}}$ morphology is intriguing since all of the molecules in the self-assembly system are achiral (see Fig. 1b,c). Therefore, a racemic mixture of chiral network domains is expected. We identified local and global chirality of ISA-H1 via structural simplification technique since 3D network structures are usually complex. Applying a surface-thinning algorithm to the reconstruction data 28,29 , we skeletonized the $3 \mathrm{D}$ networks and quantized NP network connectivity. Before application to experimental data, as an example we analysed a theoretical $G^{A}$
NP network obtained by the SCFT (see Fig. 4a). As expected for the $\mathrm{G}^{\mathrm{A}}$ morphology ${ }^{30}$, triple nodes and struts are successfully identified in the cubic unit cell and defects are found only on the artificially cleaved surface planes. A similar situation is found for the reconstructed slab where defects are focused on the edges of the slab (see Fig. 4b). However, a small number of defects can also be clearly identified within the reconstructed sample volume.

The chirality of individual gyroidal nodes was identified by calculating the angle difference between planes of neighbouring triple nodes as shown in Fig. 4 (note that the networks with $d=6.5 \mathrm{~nm}$, green networks in Fig. 3, were used for identifying the chirality since the network connectivity barely changes above this value, see Supplementary Fig. 2). As the histogram in Fig. 4d shows, most gyroidal nodes have a clockwise rotation, that is, positive angle, and the largest population is at $\theta \approx 70.5^{\circ}$, which is the theoretically expected value for gyroidal nodes ${ }^{22}$. Since counter-clockwise rotations seem to come mostly from selfassembly defects as well as artificial defects from the thin sample preparation, the sample is effectively a single chiral domain. Point, line and plane defects are observed, while chiral domain boundaries are not identified in the $3 \mathrm{D}$ data set (see Fig. 4b,c). Although only a submicron sample area was characterized, one can expect large (several tens of microns) chiral domain formation, as recently observed in the far-field optical characterization of BCP and electrodeposition-derived $\mathrm{G}^{\mathrm{A}}$ metamaterials $^{26}$, where circularly polarized light propagation was observed as predicted for chiral domains ${ }^{31}$.

Ligand density effect on nanoparticle superstructure. Having established the local and global structural characteristics of the $\mathrm{G}^{\mathrm{A}}$ hybrid, for a better understanding of the underlying physics of this self-assembly system, we quantitatively compared the experimental results with predictions from a SCFT approach, a field theoretic approach that enables predictions of self-assembled materials morphology at the mesoscale, usually challenging for particle-based simulations ${ }^{16,23,32-34}$. To that end, we recently developed a theory that allowed study of $\mathrm{ABC}$ terpolymer/ligandstabilized NP self-assembly and predicted $\mathrm{G}^{\mathrm{A}} \mathrm{NP}$ network formation, where the $\mathrm{C}$ block had affinity to ligand-stabilized NPs thus corresponding to PDMAEMA in the current experiments $^{16,17}$. For direct comparison, 2D NP distributions, projected along the (111) direction, were calculated for the $3 \mathrm{D}$ reconstruction data (Fig. 5b) and from results of two different SCFT simulations (Fig. $5 c, e$ ). As a reference system, one of the simulated systems in Hur et al. ${ }^{17}$ was chosen; see results in Fig. 5b (see Methods section and Supplementary Fig. 4 for a more detailed description). A more dispersed NP distribution is observed in the reference system than in the experiment (compare Fig. 5b,c). To better account for the true experimental structure of the ligand-stabilized NPs $\left(N_{\mathrm{L}}=60.8\right.$ and branched ligand), the number of ligands per NP was increased by a factor of 3 , to $N_{\mathrm{L}}=60$, and ligands were made two times larger ${ }^{35}$. The resulting simulated NP density distribution in Fig. $5 \mathrm{e}$ is almost identical to the one found experimentally (Fig. 5b).

Owing to a larger effective volume, NPs with more and larger ligands tend to be more aggregated to the centres of gyroidal triple nodes and struts (see Fig. 5a for structural details of gyroidal triple nodes and struts projected along (111) direction) similar to large NPs segregating to the middle of lamellae in a $1 D$ lamellar morphology 34 . Entropic driving forces of mixing for larger molecular objects are weak. Thus, enthalpic driving forces dominate self-assembly and induce NPs to be more separated away from enthalpically repulsive blocks (see illustrations in Fig. 5d,f). Other experimental factors have much weaker effects on NP distribution than the ligands (see Supplementary Fig. 4). 

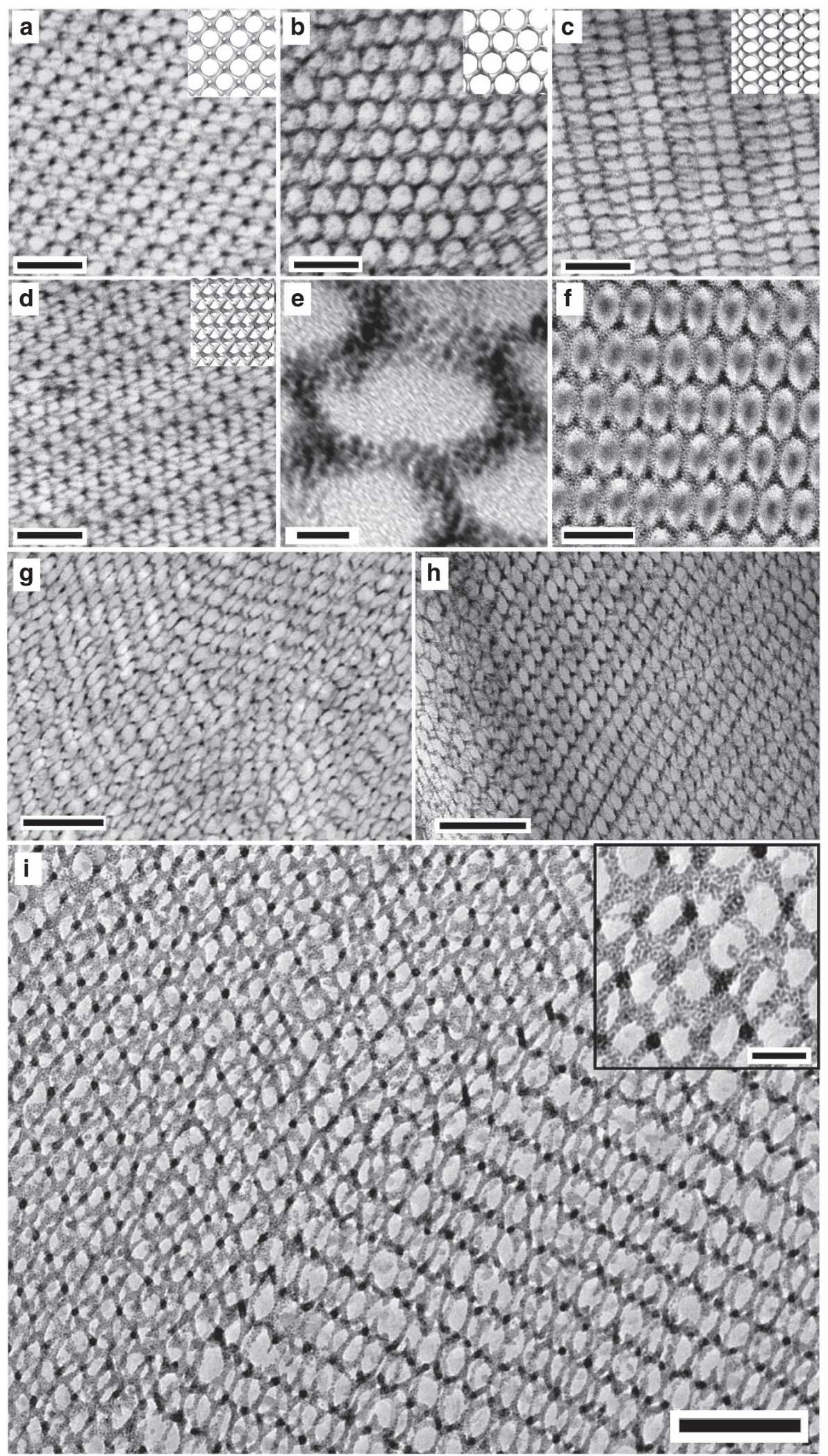

Figure 2 | TEM characterization of materials. Representative TEM images of ISA-H1 (a-f), ISA-H2 (g) and ISA-H3 (h) before removal of organic materials. ISA-H1 film with about $100 \mathrm{~nm}$ thickness was further treated with Ar-O plasma for 20 min and its TEM image is shown in i. (a-f) ISA-H1 with different projections: (a), (100); (b), (111); (c), (110); (d), (210); (e), higher magnification image showing individual Pt NPs; (f), (111) projection with PI domain stained by $\mathrm{OsO}_{4}$. Insets show SCFT simulations of expected contrast distributions for different projections; hybrids with binary Pt and Au NP mixtures, (g) ISA-H2 and (h) ISA-H3. (i) ISA-H1 after Ar-O plasma treatment at different magnifications (inset). Scale bars, $100 \mathrm{~nm}$ (a-d), $10 \mathrm{~nm}$ (e), $50 \mathrm{~nm}(\mathbf{f}), 200 \mathrm{~nm}(\mathbf{g}, \mathbf{h}), 100 \mathrm{~nm}(\mathbf{i}), 20 \mathrm{~nm}$ (inset of $\mathbf{i}$ ). 


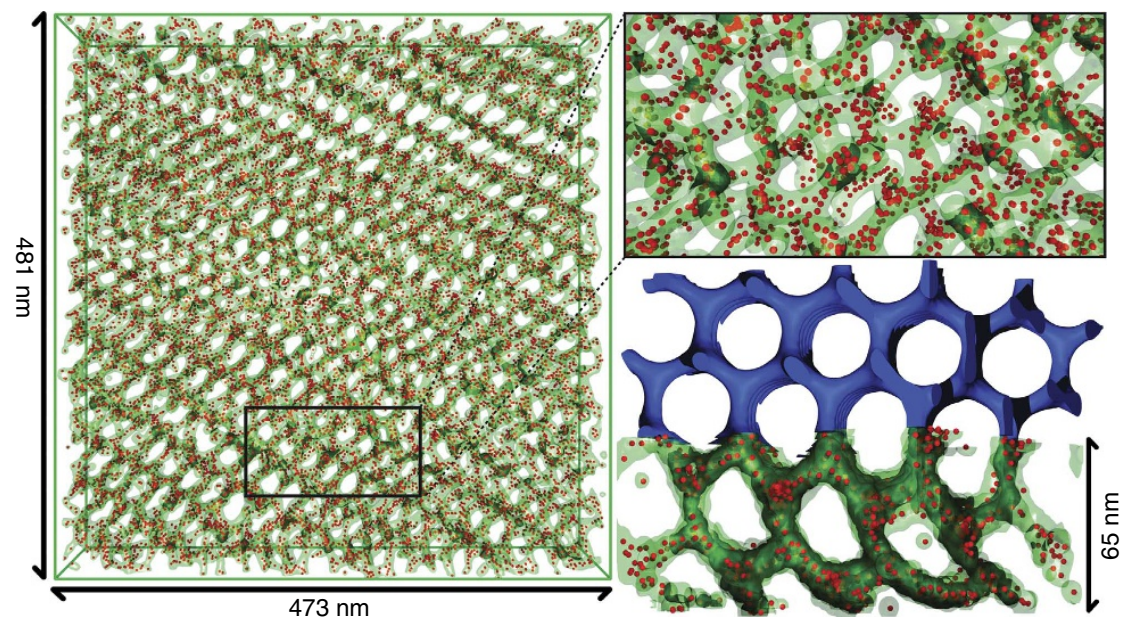

Figure 3 | TEMT reconstruction of Pt NP network superstructure in as-made ISA-H1 and comparison with SCFT results. The sample was imaged without the plasma treatment. Red spheres are Pt NPs and green volumes represent networks formed by enlarging the radii of NP cores. On the lower right a blue gyroid network with (111) projection obtained by a SCFT approach is superimposed for comparison.

a

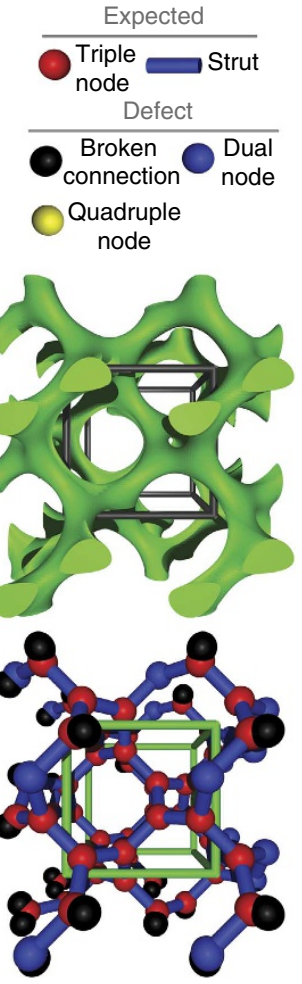

b
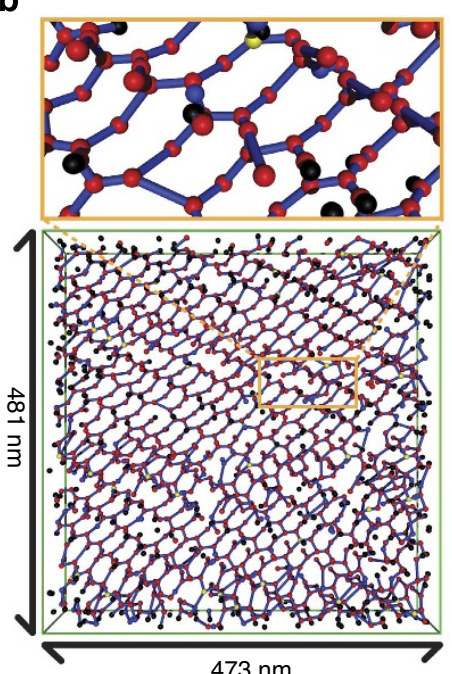

C
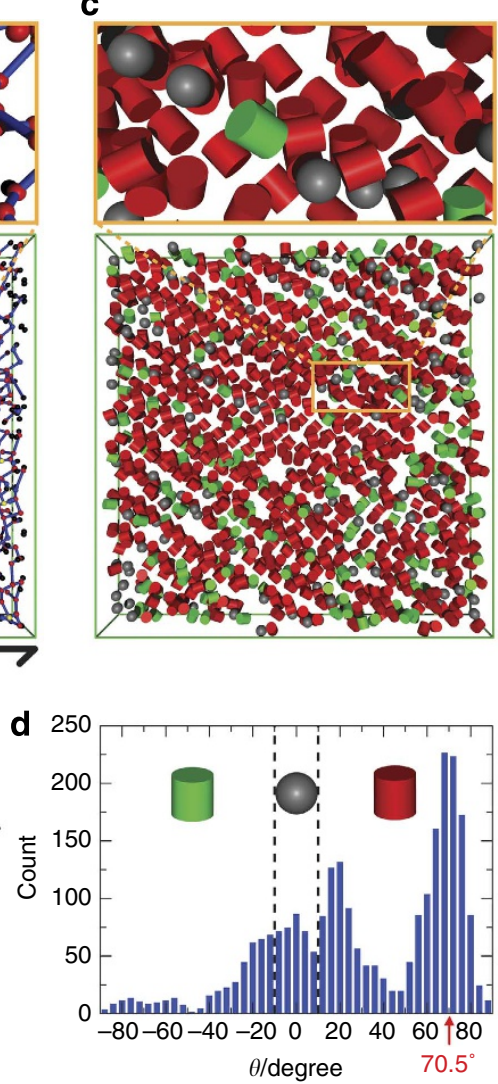

Figure 4 | Identification of local and global contributions to structural chirality in gyroidal networks of ISA-H1. (a) A $2 \times 2 \times 2$ cubic unit cell of a chiral $\mathrm{G}^{\mathrm{A}} \mathrm{NP}$ network obtained from SCFT simulation (top); nodes and struts identified via a surface-thinning algorithm (bottom) ${ }^{28}$. The black and green boxes represent a cubic unit cell in the simulated and thinned structures, respectively. For the $\mathrm{G}^{\mathrm{A}}$ network, triple nodes and struts can be identified, for example, inside of the green cubic unit cell ${ }^{30}$ and defects are found only on artificially cleaved planes. (b) Network connectivity of NPs obtained by the surface-thinning algorithm, where NP core diameters are enlarged to $d=6.5 \mathrm{~nm}$ (green networks in Fig. 3 ) and the same colour codes as in a are used. Thin top and bottom layers, about one-sixth of the sample for each layer, are not shown because defects in the layers were generated during the thin hybrid sample preparation. (c) Chirality of each node is identified from rotation angles of neighbouring gyroidal node planes, $\theta$; see model. Red cylinders correspond to clockwise $\left(\theta=10^{\circ} \sim 90^{\circ}\right)$, green cylinders to counter-clockwise $\left(\theta=-10^{\circ} \sim-90^{\circ}\right)$ and grey spheres to other rotation angles $\left(\theta=-10^{\circ} \sim 10^{\circ}\right)$. Cylinder axes represent directions normal to gyroidal node plane. (d) Histogram for experimental rotation angles. The largest population is observed at $70.5^{\circ}$, which is the theoretically expected value for gyroidal nodes. 


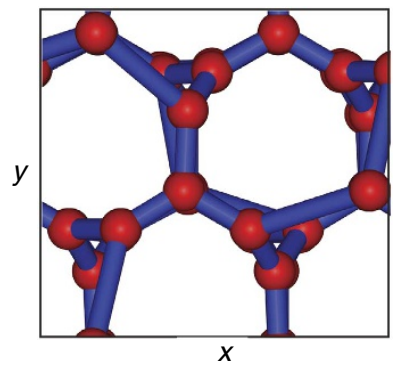

g

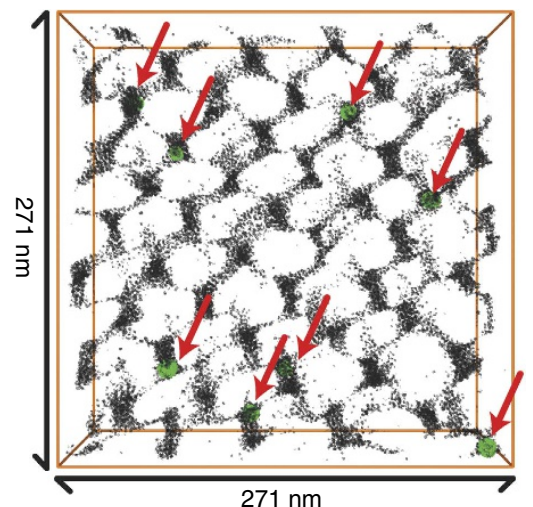

b

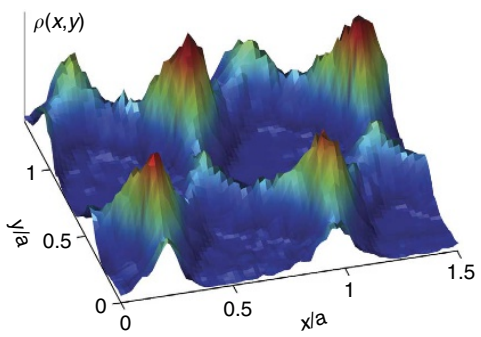

h

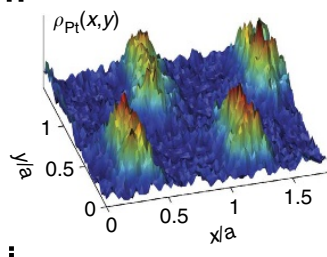

j

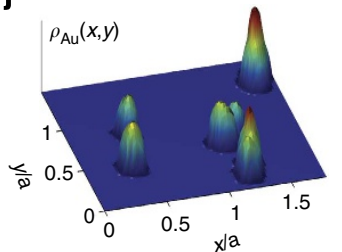

C $\rho(x, y)$

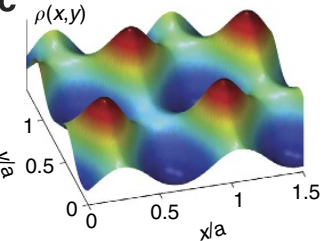

$\mathbf{e}$

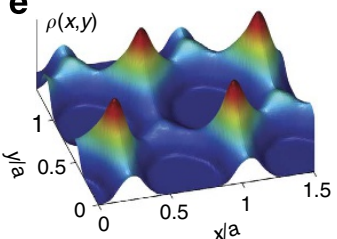

i $\mid \rho_{\mathrm{Pt}}(x, y)$

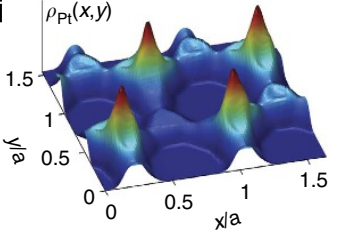

k

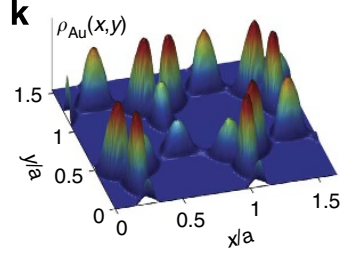

d

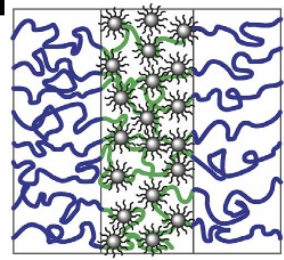

f

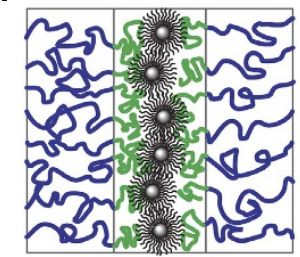

\section{I}

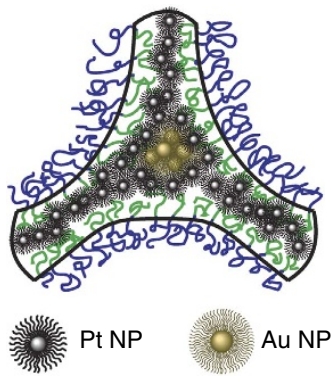

Figure 5 | Quantitative comparison of NP locations between experiment and theory. 3D data analyses of (b-f) single and (g-I) binary metal NP systems. NP distribution profiles projected along (111) direction, $\rho(x, y)$, were compared between (b,h,j) experiments and (c,e,i,k) SCFT simulations: (a) (111) projection of gyroidal nodes (red spheres) and struts (blue cylinders) of $\mathrm{G}^{\mathrm{A}}$; (b) Pt NPs of ISA-H1; (c) NPs of Hur et al. ${ }^{17}$; (d) illustration of NP distribution of c in a strut; $(\mathbf{e})$ NPs with three times more $\left(N_{\mathrm{L}}=60\right)$ and two times larger ligands than those in $\mathbf{c}$; (f) illustration of NP distribution of $\mathbf{b}$ and $\mathbf{e}$ in a strut; (g) TEMT and EDS elemental mapping of Pt (black) and Au (green) NPs in the polymer-containing hybrid ISA-H4, where Au NPs are indicated by red arrows; (h) Pt NPs of ISA-H4; (i) Pt NPs of SCFT simulation; (j) Au NPs of ISA-H4; (k) Au NPs of SCFT simulation; and (I) illustration of NP distribution of $\mathbf{h}-\mathbf{k}$ in a gyroidal node. Note that $a$ in $\mathbf{b}, \mathbf{c}, \mathbf{e}$ and $\mathbf{h}-\mathbf{k}$ is the cubic lattice parameter.

The radial distribution function of Pt NPs as a function of NP to NP distance, $r$, in Fig. 6a, as obtained from analysis of the reconstruction data, details both short- and long-range NP-NP correlations. The maximum is observed at $r \approx 3 \mathrm{~nm}$, much smaller than the NP diameter $(\sim 5 \mathrm{~nm})$ including the ligand layer (3$3.6 \mathrm{~nm}$; note that the average NP core diameter is about $1.8 \mathrm{~nm}$ (ref. 35)). This suggests that the majority of the NPs are interdigitated. Close inspection of the reconstruction data reveals that NP ligands are aggregated mostly in the triple nodes, while NPs in the struts are mostly disconnected. The SCFT simulation reveals the highly aggregated regime (triple nodes) and the sparse NP regime (struts) as shown in Fig. 6b. The aggregation in triple nodes is experimentally corroborated via long-range correlation peaks of the radial distribution function that coincide with distances from a node to a nearest neighbour $(r=21 \mathrm{~nm})$ and a second nearest-neighbour node $(r=39 \mathrm{~nm})$. This corresponds to a cubic lattice dimension of $a=59 \mathrm{~nm}$, close to the value of $63 \mathrm{~nm}$ as measured by SAXS (see the inset of Fig. 6a).

Control of binary metal NPs superstructure. The ligand density on the Pt NP surface is a key parameter for governing spatial arrangement of the NPs in this self-assembly system. Moving a step further, we hypothesized that ligand chemistry may control the NP spatial arrangement in binary NP-based 3D network superstructures. The hypothesis was tested via synthesis of a metal NP with higher ligand density than observed for the Pt NPs. For controlled experiments, a newly synthesized NP should have similar NP metal core diameter but should be distinguishable from the Pt NP in an electron microscope. We achieved these requirements by choosing $\mathrm{Au}$ and a linear molecule as NP metal core and ligand, respectively. A linear ligand is expected to yield higher ligand density on NP surface as compared with a branched ligand because of less steric repulsion between the ligands (see Fig. 1c). Resulting Au NPs had similar metal core diameters but a ligand density 3.5 times as high as for Pt NPs (see Supplementary Fig. 5).

We prepared binary NP hybrids from 18 to $20 \mathrm{wt} \% \mathrm{Pt}$ and $\mathrm{Au}$ NPs, with 5,10 and $15.8 \mathrm{wt} \%$ of the total NPs being Au, referred to as ISA-H2, ISA-H3 and ISA-H4, respectively (see Table 1). With increasing Au NP content, broadening of SAXS peaks was observed, indicating a more disordered lattice, along with a systematic lattice parameter increase in Fig. 1e. On the basis of these SAXS traces alone, structure assignments were not possible. TEM micrographs in Fig. 2g,h clearly established metal NP-based single networks for both ISA-H2 and ISA-H3. Thus, while maintaining a 3D NP superstructure, binary metal NPs composition can be programmed.

A combination of TEMT and EDS provided independent spatial distribution of $\mathrm{Pt}$ and Au NPs (see Fig. 5g,h,j and Supplementary Fig. 6). Owing to the loss of long-range order as evidenced by the SAXS measurements in Fig. 1e, NP distribution of the binary system projected along (111) direction is less clear 
a

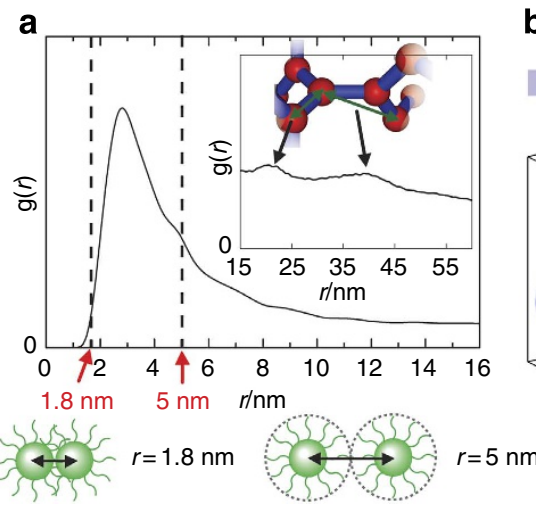

Figure 6 | Short- and long-range NP-NP correlations. (a) Radial distribution function, $g(r)$, of NPs as a function of distance, $r$, from tomographic data set of ISA-H1. The inset details long-range correlations in the data. (b) Isosurfaces of NP density from the SCFT simulation of Fig. 4c at three different densities (a.u.). Highly concentrated NP distributions are observed in triple nodes, shown as red spheres in the inset of $\mathbf{a}$. The cubic lattice parameter, $a=59 \mathrm{~nm}$, based on distances from a node to a nearest neighbour $(r=21 \mathrm{~nm})$ and a second nearest-neighbour nodes $(r=39 \mathrm{~nm})$ is close to the value of $63 \mathrm{~nm}$ obtained from SAXS on the experimental system.

than that of the Pt NP system (compare Fig. 5b with h). Interestingly, Au NPs are strongly localized only in gyroidal nodes (see green spheres indicated by red arrows in Fig. 5g and NP distribution in Fig. 5j). To account for this phenomenon, we performed SCFT simulations of binary ligand-stabilized NP systems, where we used $N_{\mathrm{L}}=210$ for Au NPs to match the experimental conditions. However, such unusual localization of Au NPs to gyroidal nodes was not initially observed in the SCFT simulation (see Supplementary Fig. 7). Simulations with strong enthalpic repulsions between B blocks (PS block in experiment) and $\mathrm{Au}$ NPs, where interaction parameter, $\chi_{\mathrm{B}, \text { Ligand }} N$, was changed from 14.2 to 40 , reproduced such behaviour (compare Fig. 5k with Supplementary Fig. 7b). To minimize the enthalpic energy penalty, Au NPs more repulsive to the B blocks than Pt NPs dominantly occupy gyroidal triple nodes (see Supplementary Fig. 8). Strong enthalpic repulsions between PS blocks and Au NP ligands are readily expected owing to the terminal hydroxyl group of the Au NP ligand (see Fig. 1c). As for the case of Pt NPs only, binary NP hybrids could be treated with Ar-O plasma, resulting in mesoporous materials (see Supplementary Fig. 9).

These results suggest that NP ligands dominantly govern the BCP/NP self-assembly. Careful design of NP ligands in terms of ligand density and interactions between NPs and BCPs can enable programmable control of the NP spatial arrangement in such 3D self-assembly systems. Interestingly, a recent study on superstructure formation in semiconductor quantum dot self-assembly also elucidated that ligand density and molecular structure are the key parameters governing NP assembly despite very distinct molecular structures ${ }^{36}$.

In summary, we presented the preparation and analysis of highly ordered 3D single-network superstructures of single and binary metal NPs from triblock terpolymer/NP self-assembly. Plasma etching of the hybrids, both with Pt NPs and with Pt and $\mathrm{Au}$ NPs, resulted in porous materials without the loss of NP superstructure. Structures were studied at an unprecedented level of detail utilizing a combination of TEMT, EDS, SCFT and percolation analysis to understand the underlying physics of selfassembled NP superstructure formation. Analysis of NP radial distribution elucidated significant NP ligand interdigitation as well as long-range correlations owing to clustering of NPs in gyroidal nodes. We demonstrated how application of surfacethinning algorithms to the tomographic data allows detailed analysis of local and global contributions to structural chirality of the $\mathrm{G}^{\mathrm{A}}$ network. Finally, we found that careful design of ligand structure in binary metal NP terpolymer assembly can enable programmable control of the NP spatial arrangement in 3D single-network superstructures owing to its dominant effects on NP distribution. We expect that results of this study will help guide future fabrication of porous NP-based network superstructures for applications ranging from high charge density electrodes to high surface area catalysis.

\section{Methods}

Triblock terpolymer preparation. PI- $b$-PS- $b$-PDMAEMA (ISA, Fig. 1b) was synthesized via sequential anionic polymerization ${ }^{37}$. As far as we know, this is the first report on the synthesis and properties of this terpolymer. Aliquots of samples were taken after each polymerization step for analysis. Figure 1d shows the size exclusion chromatography results for PI, PI- $b$-PS and the final triblock terpolymer PI- $b$-PS- $b$-PDMAEMA, demonstrating well-controlled polymerizations with narrow molar mass distributions (poydispersity index $<1.1$ ) after each step. The accurate molar mass of the first PI block was determined by size exclusion chromatograph against a PI calibration, while the final triblock terpolymer composition and molar mass was calculated based on ${ }^{1} \mathrm{H}$ NMR integration (see Supplementary Fig. 1a) and the accurate PI molar mass. The final terpolymer had molar mass of $102,300 \mathrm{~g} \mathrm{~mol}^{-1}$ and $23.4 \mathrm{wt} \%$ PI, $71.5 \mathrm{wt} \%$ PS and $5.1 \mathrm{wt} \%$ PDMAEMA.

Nanoparticle synthesis. The synthesis of the Au NPs stabilized by the ligand 11 mercapto-1-undecanol used in this study is detailed in Zheng et al. ${ }^{38}$ Ligandstabilized Pt NPs were synthesized by a synthesis scheme that is improved over what was described in our previous work ${ }^{6,35} . \mathrm{PtCl}_{4}(0.78 \mathrm{~g})$ were dissolved in $200 \mathrm{ml} \mathrm{H} 2 \mathrm{O} /$ methanol

$(1 / 1 \mathrm{v} / \mathrm{v})$ solvent mixture (solution A). Ligand $(0.30 \mathrm{~g}), N, N$-di-(2-(allyloxy)ethyl)$\mathrm{N}$-3-mercaptopropyl- $\mathrm{N}$-methylammonium chloride and $0.86 \mathrm{~g} \mathrm{NaBH}$ were taken out from the glove box and dissolved in $10 \mathrm{~g}$ methanol (ligand solution $\mathrm{B}$ ) and $15 \mathrm{~g}$ $\mathrm{H}_{2} \mathrm{O}\left(\mathrm{NaBH}_{4}\right.$ solution $\left.\mathrm{C}\right)$, respectively. Solutions B and $\mathrm{C}$ were subsequently added into solution A quickly (within $5 \mathrm{~s}$ ) while stirring vigorously. The solution colour changed from black to dark brown within $5 \mathrm{~min}$, indicating the formation of sub 3-nm NPs. The solution was further allowed to stir for $45 \mathrm{~min}$, and then $50 \mathrm{ml}$ methanol was added to dilute the solution and terminate the reaction. Methanol was rotary evaporated out and the solution was freeze dried on a vacuum line to remove excess water. The NPs were subsequently dissolved in chloroform and filtered through filter paper to remove salts formed in the synthesis. To remove residual free ligand, the particles were finally washed with a chloroform/ ethyl ether mixture for four to five times. The final Pt NPs were a black powdery solid.

Hybrid synthesis. The hybrid films were prepared via evaporation-induced self-assembly of ISA, Pt NPs and Au NPs from a common solvent. The solid components were mixed in a ratio listed in Table 1 and were dissolved in 9:1 (wt) chloroform/methanol mixture so that the total solid concentration was $10 \mathrm{wt} \%$. The solutions were poured in aluminium dishes and were heated to $50{ }^{\circ} \mathrm{C}$ on a hotplate under a hemispherical dome to slow down the evaporation. Finally, the films were heated in a vacuum oven at $130{ }^{\circ} \mathrm{C}$ to remove the solvents. The resulting films were sectioned with a Leica FC7 cryoultramicrotome to $100 \mathrm{~nm}$ for electron microscopy imaging.

Conventional TEM. Sectioned samples before and after two to four cycles of $5 \mathrm{~min}$ Ar-O plasma treatment (Fischione Model 1020) were used. TEM images were acquired in a Tecnai T12 Spirit microscope with a $\mathrm{LaB}_{6}$ source and $120 \mathrm{kV}$ acceleration voltage (Fig. 2; Supplementary Fig. 9). Scanning TEM (STEM) images with EDS data for ISA-H1 before and after plasma treatment were obtained in a Tecnai F20 FE-STEM operated at $200 \mathrm{kV}$ acceleration voltage (Supplementary Fig. 3).

TEMT and EDS. TEM samples before plasma cleaning were used for TEMT. Before TEMT of ISA-H1, Au NPs (diameter: $5 \mathrm{~nm}$ ) were placed on the supporting membrane of the TEM grid. TEMT was performed on an electron microscope operated at $200 \mathrm{kV}$ (JEM-2200FS, JEOL Co., Ltd., Japan) and equipped with a slowscan charge-coupled device camera (Gatan USC4000, Gatan Inc., USA). A series of projection images were taken from $-70^{\circ}$ to $+70^{\circ}$ at an angular interval of $1^{\circ}$. The tilt series of images were aligned by the fiducial marker method using the Au NPs as fiducial markers ${ }^{39}$. After the alignment, $3 \mathrm{D}$ images were reconstructed by the filtered back projection algorithm ${ }^{15}$. 
For TEMT and EDS of ISA-H4, the thin section was coated with carbon on the upper side. Au NPs with $5 \mathrm{~nm}$ diameter were deposited on the back side of the thin section. The specimen was tilted from $-71^{\circ}$ to $65^{\circ}$ at $1^{\circ}$ steps. The tilting series of TEM images were acquired on a JEM-2200FS operated at $200 \mathrm{kV}$. After TEMT observation, EDS measurement at same field of view was carried out on a JEMARM200F operated at $200 \mathrm{kV}$. The EDS mapping images at four fields of view were combined into one mapping image.

SAXS experiments. SAXS patterns of samples were obtained at Cornell High Energy Synchrotron Source G1 station with a beam energy of $9 \mathrm{keV}$ as well as at a home-built Rigaku RU300 CuK $\alpha$ rotating anode beamline. The details of both SAXS beamline configurations are described elsewhere ${ }^{40}$. Acquired 2D patterns were azimuthally integrated around the beam centre to produce intensity versus scattering vector magnitude, $q$, defined as $q=4 \pi \sin \theta / \lambda$, where $2 \theta$ is the total scattering angle and $\lambda$ is the X-ray wavelength.

Network connectivity identification. NPs were identified from 3D TEMT data by setting a large threshold that exceeds signals of organic components. The binary data, where the regime occupied by NP cores is 1 and otherwise 0 , were convoluted with a sphere to form networks. Using a surface-thinning algorithm ${ }^{28}$, coordinates and branching orders of gyroidal nodes as well as connectivity between the nodes were obtained.

Nanoparticle percolation. Since NPs are coated with ligands, most of the NP cores are separated as in Fig. 3 (note that the identification of organic parts of the hybrid, that is, polymers and ligands, is challenging owing to small electron density contrast between them as shown in Fig. 2a-e). Therefore, 3D superstructures are not easily identified. To identify NP superstructure, we increased NP core diameters for NP cores to overlap, thus forming continuous networks, that is, convoluted NP cores with a sphere with diameter $d$. Since the size of the ligand molecule with a fully stretched configuration is about $1.5 \sim 1.8 \mathrm{~nm}$ (Supplementary Fig. 4), with $d=3.2 \mathrm{~nm}$ for the ligand layer thickness exhibits NP networks connected by ligands. Each regime in different colours in the bottom panels of Supplementary Fig. 2 corresponds to a NP network domain where NPs are connected within the domain and disconnected with NPs outside the domain. Since many NPs are separated farther than $d=3.2 \mathrm{~nm}$ in Supplementary Fig. 2, relatively small domains are observed.

A slight increase of $d$ from the ligand layer thickness leads to formation of big domains (see the bottom panel of Supplementary Fig. 2c). Such network domain formation can be detailed by a percolation theory ${ }^{27}$. For applying percolation theory, a gyroidal network domain can be simplified as a set of nodes connected by struts as in Fig. 4a. The node-to-node percolation depends on either open or closed strut connections, where the open and closed strut connections correspond to discontinuous and continuous NPs through the strut, respectively. Finite size domains are formed below a percolation threshold (Supplementary Fig. 2a,b), while an infinitely large domain is formed above the threshold (Supplementary Fig. 2c-f). The gyroidal network confines positional freedom of NPs within PDMAEMA blocks with larger NPs having higher chances of being continuous within the strut. Therefore, the node-to-node percolation probability, $\alpha$, is approximately the volume ratio of NPs to NPs plus PDMAEMA block and estimated to be $\alpha=0.65$ for our hybrid. Utilizing the percolation theory ${ }^{27}$, we can estimate the percolation threshold $\alpha_{c}=1 /(Z-1)=0.5$, where $Z$ is the coordination number that characterizes the number of neighbouring nodes with $Z=3$ for the gyroidal triple node. Thus, our hybrid sample is above the threshold, $\alpha_{c}=0.5$. Despite a larger $\alpha$ than the threshold for the case of $d=3.2 \mathrm{~nm}$ corresponding to an extended ligand conformation, however, small domains are observed in Supplementary Fig. $2 \mathrm{~b}$, implying that a realistic $\alpha$ is close to, but smaller than, $\alpha_{c}$ owing to inhomogenous NP distribution (see the change of network connectivity and domain size as $d$ increases in Supplementary Fig. 2). Please note that the inhomogeneity is consistent with results of the radial distribution function of NPs and NP density distributions of SCFT simulations as shown in Fig. 6.

The domain size below the critical point is expected to be a few cubic unit cells, that is, $\sim 50 \mathrm{~nm}$, since the number of cubic unit cells per domain is approximately given by $1 /[1-(Z-1) \alpha] / 10$ (ref. 41) and order of 1 for $0.9<(Z-1) \alpha<0.99$ in close proximity to the critical point (note that the factor 10 comes from 10 triple nodes existing in a gyroid cubic unit cell ${ }^{30}$ ).

At the molecular level, NP ligands are interdigitated in the triple node while NPs are disconnected within the strut. Interestingly, the Pt NP superstructure was maintained after plasma treatment of hybrid ISA-H1 (see Fig. 2i), which removed organic components. The survival of the NP superstructure can be rationalized based on the shrinkage of the gyroidal network. First, 20\% decrease of gyroidal node-to-node distance is observed in the structure after the plasma treatment (compare Fig. 2e with i), accompanied with smaller NP-NP spacings in the struts. Furthermore, from high-angle annular dark-field imaging-STEM image analysis thinning of the gyroidal struts is evidenced (see Supplementary Fig. 3a,b). Therefore, the plasma-treated NP network may move above the percolation threshold without organic materials, thus maintaining the superstructure.
SCFT simulations. We used a recently developed SCFT/density functional theory approach to incorporate molecular details of ligand-stabilized Pt NPs ${ }^{16,17}$ To observe NP distribution variations for a fixed morphology, a symmetry-adopted real space basis set for the space group $\mathrm{Q}^{214}$ was used ${ }^{17}$. As a reference system, we chose one of the simulated systems discussed in Hur et al. ${ }^{17}$, where the predicted morphology is $\mathrm{G}^{\mathrm{A}}$, interaction parameter between ligand and $\mathrm{C}$ block $\chi_{\text {C,Ligand }} N=0, N_{\mathrm{L}}=20$ and NP volume fraction, $\phi_{\mathrm{P}}+\phi_{\text {Ligand }}=0.06$ (note that, owing to similar $\chi$ parameters for PDMAEMA and polyethylene oxide ${ }^{23,42}$, simulation parameters for polyethylene oxide were used for the $\mathrm{C}$ block in this work). For Fig. 5e, two times longer ligands with $N_{\mathrm{L}}=60$ than those of the reference system were used. For Au NPs of Fig. 5j,k, the same parameters of Pt NPs in Fig. 4e were used but the number of ligands per NP and interaction parameter between B block and ligands were varied as $N_{\mathrm{L}}=210$ and $\chi_{\text {B,Ligand }} N=40$.

\section{References}

1. Shenton, W., Pum, D., Sleytr, U. B. \& Mann, S. Synthesis of cadmium sulphide superlattices using self-assembled bacterial S-layers. Nature 389, 585-587 (1997).

2. Kiely, C. J., Fink, J., Brust, M., Bethell, D. \& Schiffrin, D. J. Spontaneous ordering of bimodal ensembles of nanoscopic gold clusters. Nature 396, 444-446 (1998).

3. Leunissen, M. E. et al. Ionic colloidal crystals of oppositely charged particles. Nature 437, 235-240 (2005).

4. Shevchenko, E. V., Talapin, D. V., Kotov, N. A., O’Brien, S. \& Murray, C. B. Structural diversity in binary nanoparticle superlattices. Nature 439, 55-59 (2006).

5. Kalsin, A. M. et al. Electrostatic self-assembly of binary nanoparticle crystals with a diamond-like lattice. Science 312, 420-424 (2006).

6. Warren, S. C. et al. Ordered mesoporous materials from metal nanoparticleblock copolymer self-assembly. Science 320, 1748-1752 (2008).

7. Nykypanchuk, D., Maye, M. M., van der Lelie, D. \& Gang, O. DNA-guided crystallization of colloidal nanoparticles. Nature 451, 549-552 (2008).

8. Zhao, Y. et al. Small-molecule-directed nanoparticle assembly towards stimuliresponsive nanocomposites. Nat. Mater. 8, 979-985 (2009).

9. Srivastava, S. et al. Light-controlled self-assembly of semiconductor nanoparticles into twisted ribbons. Science 327, 1355-1359 (2010).

10. Macfarlane, R. J. et al. Nanoparticle superlattice engineering with DNA. Science 334, 204-208 (2011).

11. Lin, Y. et al. Self-directed self-assembly of nanoparticle/copolymer mixtures. Nature 434, 55-59 (2005).

12. Cho, B.-K., Jain, A., Gruner, S. M. \& Wiesner, U. Mesophase structuremechanical and ionic transport correlations in extended amphiphilic dendrons. Science 305, 1598-1601 (2004).

13. Crossland, E. J. W. et al. A bicontinuous double gyroid hybrid solar cell. Nano Lett. 9, 2807-2812 (2008).

14. Orilall, M. C. \& Wiesner, U. Block copolymer based composition and morphology control in nanostructured hybrid materials for energy conversion and storage: solar cells, batteries, and fuel cells. Chem. Soc. Rev. 40, 520-535 (2011).

15. Jinnai, H., Spontak, R. J. \& Nishi, T. Transmission electron microtomography and polymer nanostructures. Macromolecules 43, 1675-1688 (2010).

16. Hur, K., Hennig, R. G., Escobedo, F. \& Wiesner, U. Mesoscopic structure prediction of nanoparticle assembly and coassembly: theoretical foundation. J. Chem. Phys. 133, 194108-194119 (2010).

17. Hur, K., Hennig, R. G., Escobedo, F. A. \& Wiesner, U. Predicting chiral nanostructures, lattices and superlattices in complex multicomponent nanoparticle self-assembly. Nano Lett. 12, 3218-3223 (2012).

18. Luzzati, V. \& Spegt, P. A. Polymorphism of lipids. Nature 215, 701-704 (1967)

19. Lindblom, G. \& Rilfors, L. Cubic phases and isotropic structures formed by membrane lipids-possible biological relevance. Biochim. Biophys. Acta 988, 221-256 (1989).

20. Schoen, A. H. Infinite Periodic Minimal Surfaces Without Self-intersections (NASA Technical Note, NASA TN D-5541. National Aeronautics and Space Administration, Washington DC, 1970).

21. Saranathan, V. et al. Structure, function, and self-assembly of single network gyroid (I4132) photonic crystals in butterfly wing scales. Proc. Natl Acad. Sci. USA 107, 11676-11681 (2010).

22. Bailey, T. S., Hardy, C. M., Epps, T. H. \& Bates, F. S. A noncubic triply periodic network morphology in poly(isoprene-b-styrene-b-ethylene oxide) triblock copolymers. Macromolecules 35, 7007-7017 (2002).

23. Tyler, C. A., Qin, J., Bates, F. S. \& Morse, D. C. SCFT study of nonfrustrated ABC triblock copolymer melts. Macromolecules 40, 4654-4668 (2007).

24. Bates, F. S. et al. Fluctuations, conformational asymmetry and block-copolymer phase-behavior. Faraday Discuss. 98, 7-18 (1994).

25. Floudas, G. et al. Poly(ethylene oxide-b-isoprene) diblock copolymer phase diagram. Macromolecules 34, 2947-2957 (2001)

26. Vignolini, S. et al. A 3D optical metamaterial made by self-assembly. $A d v$. Mater. 24, Op23-Op27 (2012). 
27. Stauffer, D. \& Aharony, A. Introduction to Percolation Theory 2nd edn (Taylor \& Francis, 1992).

28. Lee, T. C., Kashyap, R. L. \& Chu, C. N. Building skeleton models via 3-D medial surface axis thinning algorithms. CVGIP Graph. Model. Im. 56, 462-478 (1994).

29. Jinnai, H., Watashiba, H., Kajihara, T. \& Takahashi, M. Connectivity and topology of a phase-separating bicontinuous structure in a polymer mixture: direct measurements of coordination number, inter-junction distances and Euler characteristic. J. Chem. Phys. 119, 7554-7559 (2003).

30. Epps, T. H. et al. Ordered network phases in linear poly(isoprene-bstyrene-b-ethylene oxide) triblock copolymers. Macromolecules 37, 8325-8341 (2004).

31. Hur, K. et al. Three-dimensionally isotropic negative refractive index materials from block copolymer self-assembled chiral gyroid networks. Angew. Chem. Int. Ed. 50, 11985-11989 (2011).

32. Matsen, M. W. \& Schick, M. Stable and unstable phases of a diblock copolymer melt. Phys. Rev. Lett. 72, 2660 (1994).

33. Drolet, F. i. \& Fredrickson, G. H. Combinatorial screening of complex block copolymer assembly with self-consistent field theory. Phys. Rev. Lett. 83, 4317 (1999).

34. Thompson, R. B., Ginzburg, V. V., Matsen, M. W. \& Balazs, A. C. Predicting the mesophases of copolymer-nanoparticle composites. Science 292, 2469-2472 (2001).

35. Li, Z. et al. Metal nanoparticle, block copolymer composite assembly and disassembly. Chem. Mater. 21, 5578-5584 (2009).

36. Choi, J. J. et al. Controlling nanocrystal superlattice symmetry and shapeanisotropic interactions through variable ligand surface coverage. J. Am. Chem. Soc. 133, 3131-3138 (2011)

37. Creutz, S., Teyssie, P. \& Jerome, R. Living anionic homopolymerization and block copolymerization of (dimethylamino)ethyl methacrylate. Macromolecules 30, 6-9 (1997).

38. Zheng, N., Fan, J. \& Stucky, G. D. One-step one-phase synthesis of monodisperse noble-metallic nanoparticles and their colloidal crystals. J. Am Chem. Soc. 128, 6550-6551 (2006).

39. Luther, P. K., Lawrence, M. C. \& Crowther, R. A. A method for monitoring the collapse of plastic sections as a function of electron dose. Ultramicroscopy $\mathbf{2 4}$, 7-18 (1988).

40. Finnefrock, A. C., Ulrich, R., Toombes, G. E. S., Gruner, S. M. \& Wiesner, U. The plumber's nightmare: a new morphology in block copolymer-ceramic nanocomposites and mesoporous aluminosilicates. J. Am. Chem. Soc. 125 13084-13093 (2003)

41. Flory, P. J. Principles of Polymer Chemistry (Cornell University Press, 1953) 42. Costa, A. C., Geoghegan, M., Vlček, P. \& Composto, R. J. Block copolymer adsorption from a homopolymer melt to silicon oxide: effects of nonadsorbing block length and anchoring block, substrate interaction. Macromolecules 36, 9897-9904 (2003).

\section{Acknowledgements}

This work was supported by the NSF (DMR-1104773). K.H. was supported by award number KUS-C1-018-02, made by King Abdullah University of Science and Technology. Research made use of the Cornell Center for Materials Research Shared Facilities, supported through the NSF Materials Research Science and Engineering Centers program. The X-ray equipment was supported by Department of Energy Grant DE-FG0210ER46693. Cornell High Energy Synchrotron Source was supported by the NSF and NIH-NIGMS via DMR-0936384. H.J. gratefully acknowledges the financial support received through a Grant-in-Aid (number 24310092) from the Ministry of Education, Culture, Sports, Science, and Technology (MEXT) of Japan. We gratefully acknowledge Joerg Werner (Cornell University) for experimental assistance.

\section{Author contributions}

Z.L. prepared the hybrid films and performed TEM experiments. K.H. and H.S. sup ported the experiments in part. H.S. and S.M.G. generated and analysed the SAXS data. T.H., A.T. and H.J. performed TEMT and EDS elemental analysis. K.H. performed SCFT simulations and analysed the tomography data. K.H., Z.L. and U.W. wrote the paper. All authors commented on the manuscript. U.W. supervised the Cornell part of the work.

\section{Additional information}

Supplementary Information accompanies this paper at http://www.nature.com/ naturecommunications

Competing financial interests: The authors declare no competing financial interests.

Reprints and permission information is available online at http://npg.nature.com/ reprintsandpermissions/

How to cite this article: Li, Z. et al. Linking experiment and theory for threedimensional networked binary metal nanoparticles-triblock terpolymer superstructures. Nat. Commun. 5:3247 doi: 10.1038/ncomms4247 (2014). 